
\title{
28 Research Square \\ Identification and analysis of KLF13 variants in patients with congenital heart disease
}

\section{Wenjuan Li}

Shanghai Jiaotong University School of Medicine Xinhua Hospital

\section{Baolei Li}

Shanghai Jiaotong University School of Medicine Xinhua Hospital

\section{Tingting Li}

Shanghai Jiaotong University School of Medicine Xinhua Hospital

\section{Ergeng Zhang}

Shanghai Jiaotong University School of Medicine Xinhua Hospital

\section{Qingjie Wang}

Shanghai Jiaotong University School of Medicine Xinhua Hospital

\section{Sun Chen}

Shanghai Jiaotong University School of Medicine Xinhua Hospital

Sun Kun ( $\nabla$ sunkun@xinhuamed.com.cn )

Shanghai Jiaotong University School of Medicine Xinhua Hospital https://orcid.org/0000-0002-05047372

\section{Research article}

Keywords: congenital heart disease, KLF13, variants, TBX5, target sequencing

Posted Date: November 7th, 2019

DOI: https://doi.org/10.21203/rs.2.16986/v1

License: (c) (1) This work is licensed under a Creative Commons Attribution 4.0 International License.

Read Full License

Version of Record: A version of this preprint was published at BMC Medical Genetics on April 15th, 2020. See the published version at https://doi.org/10.1186/s12881-020-01009-x. 


\section{Abstract}

Background: The protein Kruppel-like factor 13 (KLF13) is a member of the KLF family that has been identified as a novel cardiac transcription factor which is involved in heart development. However, the relationship between KLF13 variants and CHDs in humans remains largely unknown. The present study aimed to screen the KLF13 variants in CHDs patients and genetically analyze the function of these variants.

Methods: KLF13 variants were sequenced in a cohort of $309 \mathrm{CHD}$ patients and population-matched healthy controls $(n=200)$ using targeted sequencing. To investigate the effect of variants on the functional ability of the KLF13 protein, the expressions and subcellular localization of protein, as well as the transcriptional activities of downstream genes and physically interacted with other transcription factor were assessed.

Results: Two novel heterozygous variants, c.487C>T (P163S) and c.467G>A (S156N), were identified in two out of $309 \mathrm{CHDs}$ patients with Tricuspid-valve atresia and transposition of the great arteries, respectively. No variants were found among healthy controls. The variant c.467G>A (S156N) increased protein expression and enhanced functionality compared with that of wild-type, without affecting the subcellular localization. The other variant, c.487C > T (P163S), did not show any abnormalities in protein expression and subcellular localization, however it eliminated the transcriptional activities of downstream target genes and physically interacted with TBX5, another cardiac transcription factor.

Conclusion: Our results show that the S156N and P163S variants contributed to CHD etiology. Additionally, our findings suggest that KLF13 may be a potential gene contributing to congenital heart disease.

\section{Background}

Congenital heart disease (CHD) is a term that refers to any anatomical defect in the heart or major blood vessels that is present at birth. CHD affects $1 \%$ of newborn babies, and approximately one to two per 1,000 newborn babies have critical CHDs that can cause death during the neonatal period, such as pulmonary atresia and transposition of the great arteries ${ }^{[1-4]}$. Infants and children with CHDs often suffer from delays in growth and serious progressive cardiac complications ${ }^{[5]}$. Despite significant advances in our understanding of cardiac morphogenesis, emerging evidence suggests that genetic risk factors play a crucial role in the etiology of CHD. Further elucidation of the molecular and genetic intricacies of CHDs will likely be an important step in improving treatments for CHD patients.

The heart is the first functional organ to develop during vertebrate embryogenesis, this is a complex process where multiple cell lineages contribute to organ development. The epithelial-mesenchymal transformation (EMT) is an important step during heart development, which is regulated by myocardial signals to generate mesenchymal cells that migrate into the cardiac jelly and proliferate to cellularize the endocardial cushions ${ }^{[6-7]}$. Malformation of the atrioventricular-canal cushions could lead to 
atrioventricular-septal defects (AVSDs) or other endocardial-cushion defects. Kruppel-like factor 13 (KLF13) protein has been identified as a novel collaborator with cardiac GATA4 and TBX5 factors that have high expression in the endocardium and myocardium during embryonic development. Knocking down KLF13 alleles in Xenopus embryos leads to valve immaturation, septal defects, and hypotrabeculation ${ }^{[8-10]}$. However, the mechanisms of KLF13 dysfunction causing CHDs are still poorly understood.

A spatiotemporal-expression analysis of KLF13 in murine hearts of mice showed that the earliest sign of expression at E9.5, and subsequent expression in the developing atrial myocardium, ventricular trabeculae, AV cushions, and truncus arteriosus, decreased in the postnatal heart ${ }^{[8]}$. Additionally, in Xenopus embryos, the highest levels of KLF13 were found in the interventricular septum and the atrioventricular valves and knocking down KLF13 induced ventricular hypotrabeculation, atrial-septal defects (ASDs) and delayed AV-cushion formation [8].

KLF13 is a member of the KLF family of transcription factors that have been categorized along with the specificity protein (Sp) family of transcription factors [11]. Until now, twenty-one KLF members have been identified in humans. These transcription factors are characterized by C-termini $\mathrm{Cys}_{2} \mathrm{His}_{2}\left(\mathrm{C}_{2} \mathrm{H}_{2}\right)$ zincfinger motifs that confer preferential binding to GC/GT rich sequences in gene promoters and enhancer regions to activate or inhibit downstream target expression [12-13]. ANF and BNP are natriuretic peptides that are expressed in the heart and are developmentally regulated. Their levels rise continuously during the embryonic cardiac development in both atria and ventricles when cells differentiate into cardiomyocytes. These levels then drop after postnatal development. At the genetic level, analysis of the ANP and BNP promoters have led the characterization of key cardiac transcription factors that govern cardiac growth and differentiation ${ }^{[14]}$.Studies from Lavallee et al. revealed that KLF13 could activate the BNP promoter $[8]$.

Additionally, analysis of the combined interactions of transcription-factor variants helps us better understand how the disruption of combinatorial interactions can lead to specific congenital disabilities. TBX5 is a member of the T-box transcription factors that have crucial role in regulating early cellular commitment, differentiation, and heart development ${ }^{[15] .}$ TBX5 acts as a cooperator with other transcription factors, such as GATA4 and NKX2.5, to synergistically regulate downstream targets during cardiac development ${ }^{[16-17] .}$ Previously, we showed that KLF13 is a genetic modifier of TBX5 [10].

However, since current studies of KLF13 have focused on animal models $[8,18]$, it remains unclear whether genetic variants are involved in the mechanisms of CHDs in humans. In the present study, we identified two novel KLF13 heterozygous variants in a cohort of patients with complex CHDs and compared them with those of healthy controls to evaluate the prevalence of KLF13 variants in sporadic CHDs. Our results demonstrated that these variants altered protein expression, changed the transcriptional activation of BNP and impaired the genetic interaction of KLF13 with TBX5 to promote the incidence of CHD. 


\section{Methods}

\section{Study subjects}

In this study, we recruited a total of 309 patients with complex CHD. These patients were diagnosed by echocardiography or cardiac catheterization or underwent cardiac surgery at the Shanghai Xinhua Hospital. The patients included 191 males and 118 females (Table 1). Patientswith known syndromic CHDs or chromosomal abnormalities were excluded from our study. The controls were 200 populationmatched healthy children controls without heart disease. The study protocol was reviewed and approved by the Xinhua hospital Ethics Committee. Both parents and legal guardians of the patients and healthy controls provided signed informed consents. Subsequently, peripheral blood was collected for DNA extraction. The genomic DNA was extracted using the QIAamp DNA Blood Mini Kit (Qiagen, Germany) and was stored at $-80^{\circ} \mathrm{C}$.

\section{Target sequencing and analysis}

Genomic DNA was sequenced by target-sequencing technology using the Illumina HiSeq 2000 platform for variants in KLF13 (GenBank accession number NC_000015.10, NM_015995.3). Then, Sanger sequencing was performed to validate all the candidate variants. To evaluated the protein characteristics of nonsynonymous variants, we used SIFT (http://sift.jcvi.org/), Polyphen2 (http://genetics.bwh.harvard.edu/pph2/), and Mutation Taster (www.mutationtaster.org/). Amino-acid substitutions were used to predict the damaged variants when the score was $\leq 0.05$ in SIFT or $\geq 0.85$ in Polyphen-2. KLF13 protein sequences from Homo sapiens (human), Mus musculus (house mouse), Bos taurus (cattle), Capra hircus (goat), Pan troglodytes (chimpanzee), Xenopus laevis (frog) and Sus scrofa (swine) were downloaded from the Universal Protein (UniProt) database (http://uniprot.org/) and were aligned with Clustal X software.

\section{Plasmid construction and site-directed mutagenesis}

The KLF13 and TBX5 CDNA plasmids were purchased fromGenomeditech. Site-directed mutagenesis for the KLF13 point mutations, c.467G>A (S156N) and c.487C >T (P163S), were constructed according to the protocol provided by the Site-Directed Mutagenesis Kit (Stratagene, USA). Then, the mutated sites were confirmed with Sanger sequencing. The luciferase human B-type natriuretic peptide (BNP) promoter was constructed as previously described [19].

\section{Cell cultures and transfection}

For cell culture experiments,293T cells were used for protein extraction and immunofluorescent staining. $\mathrm{NIH} 3 \mathrm{~T} 3$ cells were used for Luciferase assays and were maintained in growth medium (Dulbecco's 
Modified Eagle Medium) supplemented with $10 \%$ fetal bovine serum and 1\% penicillin/streptomycin. Plasmids were transfected with FuGene HD (Promega, USA) according to the manufacturer's protocol, 24 hours after cells were seeded.

\section{Luciferase assays}

NIH3T3 cells were seeded onto a 24-well plate, and $600 \mathrm{ng}$ of BNP luciferase reporter vector, different dosages $(25,50,100,200$, and $300 \mathrm{ng})$ of wild-type/variant KLF13 plasmids, and pCMV-Tag2B vector with or without TBX5 plasmids were transferred with FuGene HD. The luciferase activity was measured by the Dual-Gloluciferase assay system (Promega), following the manufacturers protocol after 48 hours of transfection. Firefly luciferase activities were reported as fold activation. All the experiments were repeated at least three times in duplicate.

\section{Immunofluorescence}

For myc staining, 293T cells were harvested 48 hours after transfection with wild type-KLF13 and variants. After fixation with $4 \%$ paraformaldehyde at room temperature for 10 minutes, permeation with $0.3 \%$ TritonX-100 for 15 minutes, and blocking with $5 \%$ BSA for 1 hour, cells were incubated with the primary antibody (1:500, Rabbit Polyclonal, anti-myc, 16286-1-AP, proteintech, USA) overnight at a temperature of $4^{\circ}$, followed by incubation with the conjugated secondary antibody, anti-rabbit Alexa Fluor ${ }^{\circledR} 488$ (1:500, R37116, Life technologies, USA) at room temperature in the dark for 1 hour. DAPI (Vector Laboratories, USA) was used for nuclear staining. The images were acquired using fluorescence microscopy at 200X magnification with Image Pro Plus software.

\section{Western Blotting analysis}

The cells 293T were harvested at 48 hours after transfection with wild type-KLF13 or variants. The protein was extracted as previously described ${ }^{[15]}$. A total of $50 \mu \mathrm{g}$ of the extract was subjected to $10 \%$ SDS-PAGE and was transferred onto nitrocellulose membranes and blocked with skim milk $(5 \%)$ in TBST $(0.1 \%)$ at room temperature for 2 hours, using gentle agitation during the process. Then, the membranes were incubated with the primary antibody (myc: 1:1000, Rabbit Polyclonal, anti-myc, 16286-1-AP, proteintech, USA; GAPDH: 1:1000, Rabbit monoclonal, anti-GAPDH, ab181602, Abcam, USA) overnight at a temperature of $4^{\circ}$. Following the incubation with the primary antibody, horseradish-peroxidase-conjugated secondary antibody (1:1000, 111-005-003, Jackson Immuno Research, USA), and Immobilon Western Chemiluminescent HRP Substrate (Millipore, USA) were used to visualize the chemiluminescent immunodetection.

\section{Coimmunoprecipitation}


The cells 293T were transfected with wild type-KLF13 or variants and TBX5 plasmids using FuGene HD according to the manufacturer's guidelines. Protein extracts were incubated overnight with anti-myc antibodies (1:100, Rabbit Polyclonal, anti-myc, 16286-1-AP, proteintech, USA) coupled with magnetic beads. Bound proteins were revealed with anti-TBX5 (1:1000, Rabbit Polyclonal, anti-TBX5, 42-6500, Invitrogen, USA) antibodies by Western blotting.

\section{Statistical analysis}

Data are reported as means \pm SEMs. Two-way analysis of variance (ANOVA) was used to compare differences among groups by SPSS. A $P$ value $<0.05$ was considered to indicate statistical significance.

\section{Results}

\section{KLF13 sequencing in CHD patients}

In the study, a cohort of 309 patients with CHDs was enrolled. Additionally, a total of 200, ethnicallymatched healthy subjects were also enrolled and used as control.The baseline clinical characteristics of the 309 CHDs patients are summarized in Table 1. The entire coding region of the KLF13 gene was sequenced in all the patients with CHDs through targeted sequencing. We identified two novel heterozygous variants of KLF13 in two out of 309 patients. A transversion of proline into serline at the amino acid position 163 (Exon $1 \mathrm{c} .487 \mathrm{C}>\mathrm{T}$ P163S) was identified in one patient with TA VSD ASD. Additionally, a transversion of serline into asparagine at the first nucleotide of codon 156 of the KLF13 gene (exon1: c.467G>A S156N) was found in the D-TGA patient (Figure 1 A-D). These two patients did not have any variants in other genes sequenced, and no variants of any kind were found among the control individuals. SIFT, Polyphen2, and Mutation Taster were used to perform bioinformatic predictions on these two variants. Details of the specific sequence variants are summarized in Table 2.

\section{In silico analyses of KLF13 mutant protein}

The human KLF13 has 6,825 bp on chromosome 15 and is composed of two exons and two introns to encode 288 amino acids. Song et al. analyzed the KLF13 protein structure and revealed that it is comprised of three zinc-fingers domains (Zns), distinct transcriptional activation and repression domains, and two potent independent nuclear-localization signals (NLS) ${ }^{[12]}$. Both S156N and P163S were located in the NLS1 region that was close to the DNA-binding domain (Figure 1E). Multiple sequence alignments revealed that S156N and P163S variants were highly conserved in vertebrates (Figure 1F), indicating that these variants are important and might result in alteration of gene function.

\section{Subcellular localization and expression of KLF13 variants}


We analyzed the protein expressions of these two variants in the nucleus and cytoplasm. The determinations of subcellular localization were carried out via immunofluorescence (Figure 2A). Although both were variants located in the NLS1 region, they had an equal protein distribution in the two cellular compartments compared to those of the wild type. However, the expression of the myc-tagged-KLF13 variant, using total protein extracts prepared from the transfected 293 T cells (Figure 2B), showed that the S156N variant was distinctly higher than that of the WT- KLF13 $(P<0.05)$, while the P163S variant had a similar expression to that of the wild-type, which suggested that the $\mathrm{S} 156 \mathrm{~N}$ variant might be a gain-offunction variant.

\section{Transcriptional activity of KLF13 variant protein}

To evaluate the functional ability of the KLF13 variants to regulate downstream genes, the transcriptional activities of the variants were analyzed using a $B N P$-luciferase reporter, and cells were co-transfected with wild-type or KLF13 variants. As shown in Figure 2C, a dose-response study $(25,50,100,200$, and $300 \mathrm{ng})$ showed that, in comparison to wild-type KLF13, the P163S variant had decreased transcriptional activity. In contrast, the $S 156 \mathrm{~N}$ variant had increased transcriptional activation, which indicated that KLF13 variants had effects on $B N P$ promoter transactivation $(P<0.001)$. Therefore, the variants $S 156 \mathrm{~N}$ and P163S may affect KLF13 downstream target-gene expression during cardiogenesis, for which the variant S156N was a gain-of-function mutant, while variant P163S disrupted the function of KLF13.

\section{KLF13 variants affect physical interactions with TBX5}

Previously, we reported that KLF13 is a TBX5 cofactor, these two proteins were co-localized in several cardiac cells, and they physically and functionally interact to activate cardiac promoters synergistically [10]. Therefore, to verify the impact of KLF13 variants on physical interactions with TBX5, wild-type or variant KLF13s were co-transfected with TBX5 using the BNP-luciferase promoter. As shown in Figure 3A, when co-transfected with KLF13 and TBX5 into NIH 3 T3 cells, the BNP-luciferase promoter was activated up to 75 fold; decreased synergistic transcriptional activation was detected on variant P163S, and increased synergy was observed on variant S156N. To test whether the altered synergy was due to altered physical interactions, the binding of KLF13 wild type or variants with TBX5 were carried out by CO-IP. Figure 3B shows the analysis of the CO-IP assay. P163S showed reduced synergy with TBX5 and had a decreased physical interaction with the TBX5 protein. In contrast, S156N had a significantly increased ability to physically or functionally interact with TBX5.

\section{Discussion}

KLF13, a member of the KFL family of transcription factors, has been identified as a novel cardiac factor

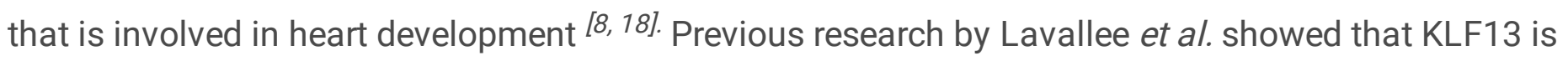
expressed during cardiac morphogenesis in mice and knocking it down induced ventricular 
hypotrabeculation, atrial septal defects (ASDs), and delayed AV-cushion formation in Xenopus embryos [8]. However, variants of KLF13 in CHD patients have remained unidentified and unelucidated. In the present study, two novels heterozygous KLF13 variants were reported using targeted sequencing in 309 patients with complex CHDs. The variant, c.467G>A (S156N), identified in the patients with TA, VSD, and ASD, had altered protein expression and functionality compared with that of the wild-type protein, without affecting the subcellular localization. The other variant, c.487C > T (P163S), found in a D-TGA patient, did not show any abnormalities in protein expression or subcellular localization. However, this variant had altered transcriptional activities of downstream genes and altered physical interactions with another cardiac factor, TBX5.

Both variants, c.467G >A and c.487C > T, were highly preserved in vertebrates based on multiple sequencealignment analyses and were located in the NLS1 region. Song et al. showed that a bipartite NLS was identified in KLF13 protein based on sequence searching, and also found that AA 147-168 (NLS1) was not the only NLS region for protein subcellular localization because deletion of this signal did not affect nuclear transport ${ }^{[12]}$. In our present results, although these two variants were located in the NLS1 region, they were also expressed in the nucleus, as was the wild-type KLF13 protein. However, the locations of these two variants were close to $\mathrm{Cys}_{2} / \mathrm{His}_{2}$ zinc-finger motifs that bind to gene promoters and enhancer regions to activate or inhibit transcription [20], suggesting that these two variants might have crucial functions in mediating the expression of downstream target genes. Lavallee et al used BNP promoter and showed that KLF13 could activate the promoter by binding to an evolutionarily conserved CACCC box [8]. Although the subcellular localization of the variant protein did not change, which is In contrast to most previously reported CHDs associated variants [21-22], we found that the $S 156 \mathrm{~N}$ variant was a gain-offunction mutation, since it significantly increased the transcriptional activity of BNP. Thus, mutation of this sites maybe may promote DNA binding activity by increasing the protein, which may well explain the up-regulated BNP in this study. Although a gain-of-function was detected in our experiments, the protein that might be less stable in vivo could also result in a loss-of-function phenotype [23-24]. Consistent with this, human heterozygous micro-deletions and duplication of the chromosomal band harboring KLF13

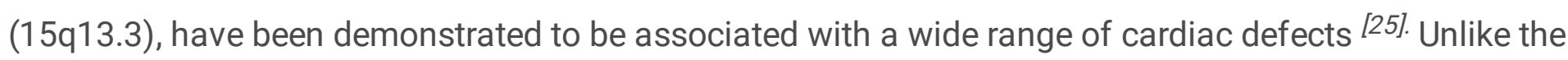
S156N variant, the $c .487 \mathrm{C}>\mathrm{T}$ variant decreased the transcriptional activity of BNP in the present study, but we did not find any disruption of expression or subcellular localization. However, a proline-to-serline substitution maybe result in impaired interactions with DNA, and the location of the P163S variant in the tertiary structure that is near the bound DNA also might lead to a decrease in the DNA-binding activity. Thus, dysregulation of downstream target genes during cardiac morphogenesis may be the reason why this variant caused TGA.

The reduced binding ability of our identified variants to targets may be primarily related to decrease DNA binding. However, structural instability, when bound to cofactors is another possibility [26]. The mechanism of cardiac transcription factors in the developing heart involves cooperative interactions with other conserved factors, and any structural defect is often linked to more than one candidate gene. Also, variable expressivity of the phenotype is often observed where CHDs are linked to a specific gene 
mutation [27]. Thus, variants of these factors may eliminate any cooperative interactions between these cofactors $^{[27-28] .}$. Previously, we showed that KLF13 and TBX5 exhibit physical and functional interactions, and that a combined heterozygous loss of TBX5 and its associated KLF13 leads to decreased postnatal viability and a higher incidence of septal defects compared to that of $T b \times 5$ heterozygous mice. Additionally, several TBX5 mutations are associated with CHDs, as indicated by impaired functional and physical interactions with KLF13, which confirms that TBX5 is a genetic modifier of KLF13 [10]. In the present study, increased transcription activity of $B N P$ was more pronounced when wild type KLF13 was co-transfected with TBX5, which indicated that KLF13 has functional interactions with TBX5 to activate BNP promoters. In our previous study of the structure-function of the TBX5-KLF13 synergy [10], the N-terminal domain of KLF13 was mainly found to support synergy with TBX5. The two novel variants of KLF13 that were detected in the $309 \mathrm{CHDs}$ patients were in the $\mathrm{N}$-terminal domain of the protein. However, the two variants had different effects on the interactions between TBX5. Significantly enhanced synergistic activity was detected in the $S 156 \mathrm{~N}$ variant, while the $\mathrm{P} 163 \mathrm{~S}$ variant eliminated this synergistic activity, which suggested that the loss of function observed in KLF13 was associated with disruption of physical and functional interactions with cofactors (TBX5), while the gain of function could enhance the interaction with cofactors.

\section{Conclusions}

KLF13 is a significant component of cardiac morphogenesis in humans. The $S 156 \mathrm{~N}$ variant identified in $\mathrm{CHD}$ patients enhanced the transcriptional function and physical interaction with the cardiac factor TBX5. In contrast, our identified P163S variant eliminated the transcriptional activities of downstream genes and reduced physical interactions with its cofactor. These findings provided genetic evidence of KLF13 dysfunction detected in CHDs based on a large population, which may provide valuable information for diagnosis and therapy in CHD patients.

\section{Abbreviations}

CHD Congenital heart disease

KLF13 Kruppel-like factor 13

EMT Epithelial-mesenchymal transformation

AVSD Atrioventricular septal defects

ASD Atrial septal defects

VSD Ventricular septal defects

TA Tricuspid valve atresia transposition of the great arteries, 
TGA Transposition of the great arteries

NLS Nuclear localization signals

Zns Zinc fingers domain

\section{Declarations}

Ethics approval and consent to participate: Research was approved by the Xinhua Hospital Ethics Committee affiliated to Shanghai Jiaotong University School of Medicine (XHEC-C-2017-105).

Consent for publication: Not applicable

Availability of data and material: The datasets used and analyzed during the current study are available from the corresponding author on reasonable request

Competing Interests: There is no competing interests.

Funding: This works was supported by grants from the National Basic Research Program of China (grant number 81700226) and the National Basic Research Program of China (grant number 81720108003).

Authors' contributions: Wenjuan Li performed all experiments and data analysis; Ergeng Zhang analyzed target sequencing data and validate all the candidate KLF13 variants; Tingting Li and Baolei Li help to perform site-directed mutagenesis and coimmunoprecipitation; Qingjie Wang provided technical assistance; Kun Sun and Sun Chen designed the experiments and wrote the paper.

Acknowledgments: The authors think Pro. Rang Xu for advice and LetPub (www.letpub.com) for its linguistic assistance during the preparation of this manuscript.

\section{References}

1. vander Linde D, Konings EE, Slager MA, Witsenburg M, Helbing WA, Takkenberg JJ, Roos-Hesselink JW .Birth prevalence of congenital heart disease worldwide: a systematic review and meta-analysis. J Am Coll Cardiol.2011.58: 2241-47.

2. Zhao QM, Ma XJ, Jia B, Huang GY. Prevalence of congenital heart disease at live birth: anaccurate assessment by echocardiographic screening. Acta Paediatr. 2013.102: 397-402.

3. Zhao QM, Ma XJ, Jia B, Huang GY. Prevalence of congenital heart disease at live birth: anaccurate assessment by echocardiographic screening. Acta Paediatr. 2013. 102: 397-402.

4. Chang RK, Gurvitz M, Rodriguez S. Missed diagnosis of critical congenital heart disease. Arch Pediatr Adolesc Med. 2008.162: 969-74

5. Cameron JW, Rosenthal A, Olson AD. Malnutrittionin hospitalized children with congenital Heart disease. Arch Pediatr Adolesc Med. 1995.149(10):1098-102. 
6. Eisenberg, L. M. and Markwald, R. R. Molecular regulation of atrioventricular valvuloseptal morphogenesis. Circ. Res. 1995.77, 1-6.

7. Anderson, R. H., Webb, S., Brown, N. A., Lamers, W. and Moorman, A. Development of the heart: (3) formation of the ventricular outflow tracts, arterial Valves, and intrapericardial arterial trunks. Heart 2003b. 89, 1110-1118

8. Lavallée G, Andelfinger G, Nadeau M, Lefebvre C, Nemer G, Horb ME et al; The Kruppel-like transcription factor KLF13 is a novel regulator of heart development. EMBO J. 2006.25(21):5201-13.

9. Nemer M, Horbi, ME.The KLF of family of transcriptional regulators in cardiomyocyte proliferation and differentiation. Cell Cycle. 2007.6(2):117-21

10. Darwich R, Li W, Yamak A, Komati H, Andelfinger G, Sun K, et al; KLF13 is a genetic modifier of the Holt-Oram syndrome gene TBX5. Hum Mol Genet.26(5):942-954

11. Lomberk, G. and R. Urrutia. The family feud: turning off Sp1 by Sp1-like KLF proteins. Biochem. J. 2005.392(1): 1-11

12. Song, A., Patel A, Thamatrakoln K, Liu C, Feng D, Clayberger $C$ et al., Functional Domains and DNAbinding Sequences of RFLAT-1/KLF13, a Krüppel-like Transcription Factor of Activated T Lymphocytes. Journal of Biological Chemistry. 2002.277(33): 30055-30065

13. Pearson, R., Fleetwood J, Eaton S, Crossley M, Bao S. Krüppel-like transcription factors: A functional family. The International Journal of Biochemistry \& Cell Biology. 2008.40(10): 1996-2001

14. Hayek S, Nemer M.Cardiac natriuretic peptides: from basic discovery to clinical practice. Cardiovasc Ther.29(6):362-76

15. Hariri F., Nemer M., Nemer G.T-box factors: insights into the evolutionary emergence of the complex heart. Ann. Med. 2012.44, 680-693

16. Garg V., Kathiriya I. S., Barnes R., Schluterman M. K., King I. N., Butler C. A., eta. GATA4 mutations cause human congenital heart defects and reveal an interaction With TBX5. Nature .2003.424, 443447

17. Hiroi Y., Kudoh S., Monzen K., Ikeda Y., Yazaki Y., Nagai R., et al. Tbx5 associates with Nkx2-5 and synergistically promotes cardiomyocyte differentiation. Nat. Genet. 2001.28, 276-280

18. Martin KM, Metcalfe JC, Kemp PR. Expression of KIf9 and Klf13 in mouse development. Mech Dev.103(1-2):149-51.

19. Zhang E, Hong N, Chen S, Fu Q, Li F, Yu Y, Sun K. Target sequencing identifies novel GATA6 variants in a large cohort of patients with conotruncal heart disease. Gene. 2018. 641:341-348

20. Toshio Nagashima, F.H., Takashi Umehara, a.S. Yokoyama.Molecular Structures of Krüppel-like Factors, in The Biology of Krüppel-like Factors, R.F. Nagai, Scott L.; Kasuga, Masato, Editor. Springer: Tokyo, Japan. 2009.268

21. Huang RT, Wang J, Xue S, Qiu XB, Shi HY, Li RG et al; TBX20 loss-of-function mutation responsible for familial tetralogy of Fallot or sporadic persistent truncus arteriosus. Int J Med Sci.14(4):323-332 
22. Yang YQ, Gharibeh L, Li RG, Xin YF, Wang J, Liu ZM et al; GATA4 loss-of-function mutations underlie familial tetralogy of fallot. Hum Mutat. 34(12):1662-71

23. Posch MG, Gramlich M, Sunde M, Schmitt KR, Lee SH, Richter S et al; A gain-of function TBX20 mutation causes congenital atrial septal defects, patent foramen ovaleand cardiac valve defects. $\mathrm{J}$ Med Genet.47(4):230-5

24. Bouveret R, Waardenberg AJ, Schonrock N, Ramialison M, Doan T, de Jong D, et al;NKX2-5 mutations causative for congenital heart disease retain functionality and are directed to hundreds of target. Elife. 2015. 6:4

25. Van Bon BW, Mefford HC, Menten B, Koolen DA, Sharp AJ, Nillesen WM, et al; Further delineation of the 15q13 microdeletion and duplication syndromes: a clinical sepectrumvarying from nonpathogenic to a severe outcome. J Med Genet. 2009. 46(8): 511-23

26. Kasinath V, Faini M, Poepsel S, Reif D, Feng XA, Stjepanovic G,et al; Structures of human PRC2 with its cofactors AEBP2 and JARID2. 2018. 359(6378):940-944

27. Nemer M. Genetic insights into normal and abnormal heart development. Cardiovasc Pathol.17(1):48-54.

28. Temsah R, Nemer M. GATA factors and transcriptional regulation of cardiac natriuretic peptide genes. Regul Pept; 2005.3:177-85

\section{Tables}

Due to technical limitations, please see the supplementary files section to access the tables.

\section{Figures}




\section{Control}

A

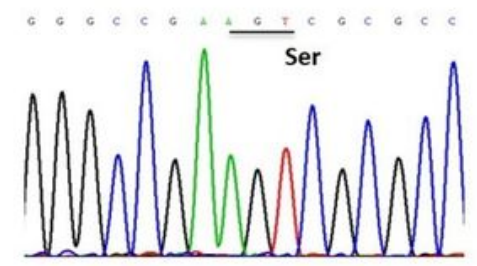

B

\section{c.467G >A p.Ser156Asn}

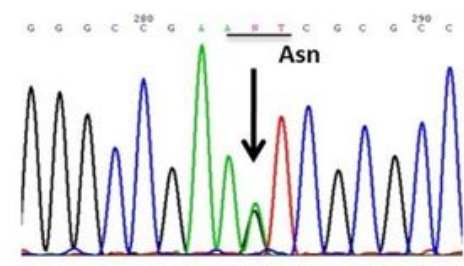

D
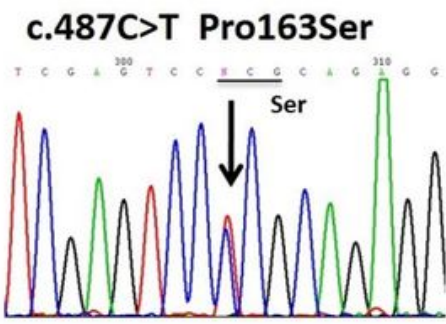

E

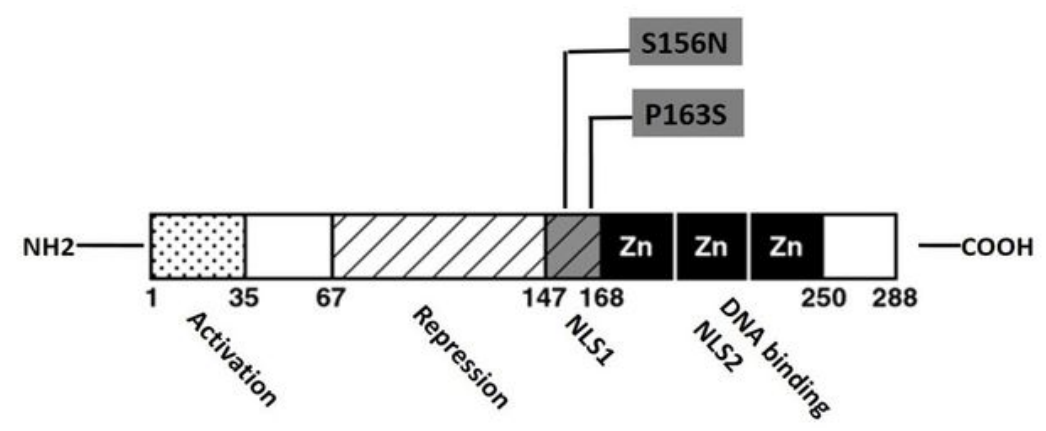

$\mathbf{F}$

KLF13

Homo sapiens

Mus musculus

Bos Taurus

Pan troglodytes

Capra hircus

Sus scrofa

Xenopus tropicalis
S156N P163S

PAGSGEPGLRQRVRRGR S RADLES P QRKHKCHYAGCEKVYGKSSHLKAHL PAGSGEPGLRQRGRRGR S RADLES P QRKHKCHYAGCEKVYGKSSHLKAHL PAGSGEPGFRQRGRRGR $S$ RTDLES P ORKHKCHYAGCEKVYGKSSHLKAHL PAGSGEPGLRQRGRRGR S RADLES P QRKHKCHYAGCEKVYGKSSHLKAHL PAGSGEPGLRQRGRRGR S RTDLES P ORKHKCHYAGCEKVYGKSSHLKAHL PAGSGEPSLRQRGRRGR S RADLES P QRKHKCHYAGCEKVYGKSSHLKAHL PPL LPEPSPKQRARR GK S RCDPES P L KKHKCPYSGCEKVYGKSSHLKAHL

\section{Figure 1}

Sequence chromatograms of KLF13 missense variants in patients and controls. A and C: Chromatograms of normal controls. B and D: Chromatograms of the three heterozygous variants. Arrows show heterozygous nucleotide changes; E: Location of the novel KLF13 variants (highlighted in bold). NLS, nuclear-localization signals; Zn, zinc finger domain. F: Alignments of KLF13 protein among different species indicated that S156N and P163S were conserved among vertebrates. 
A

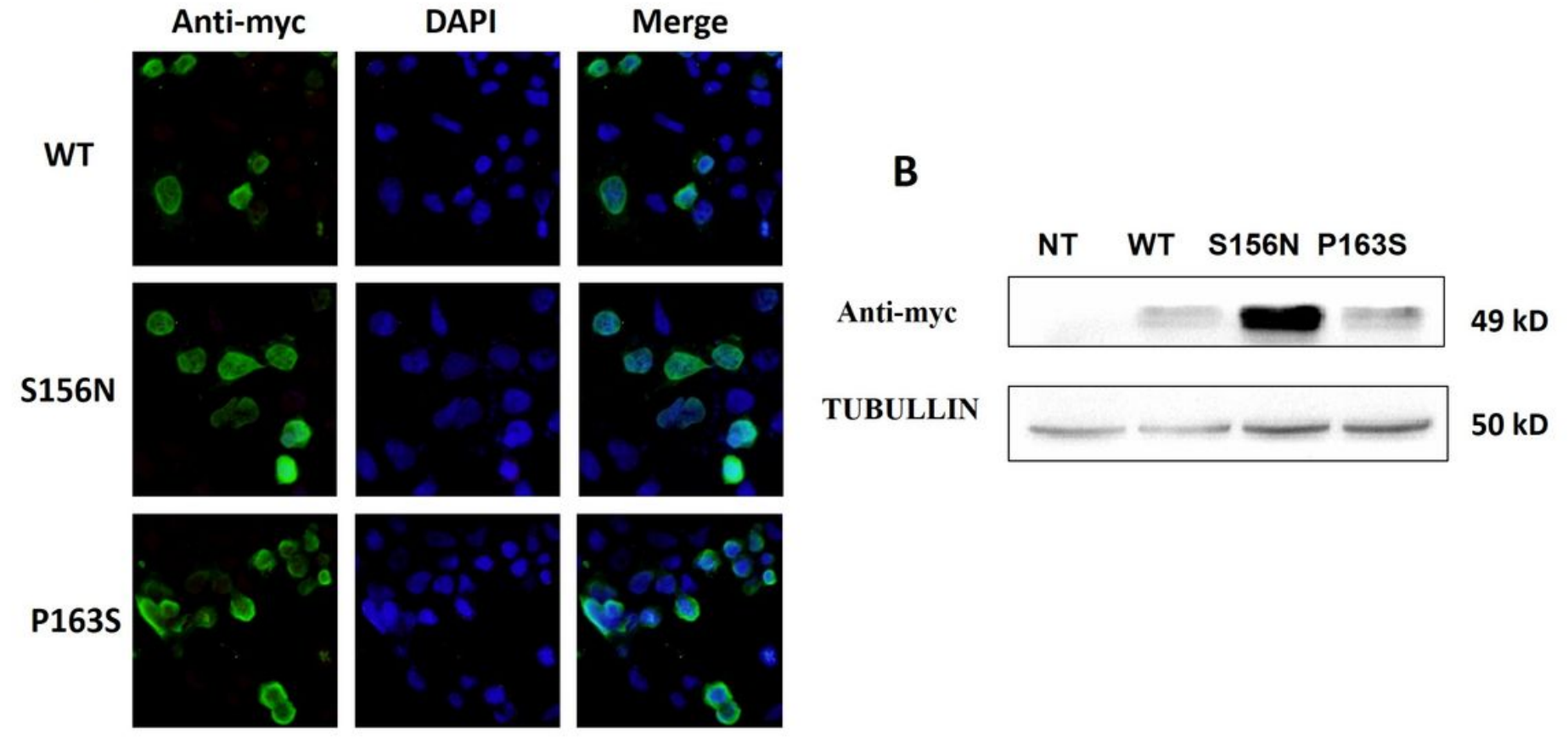

C

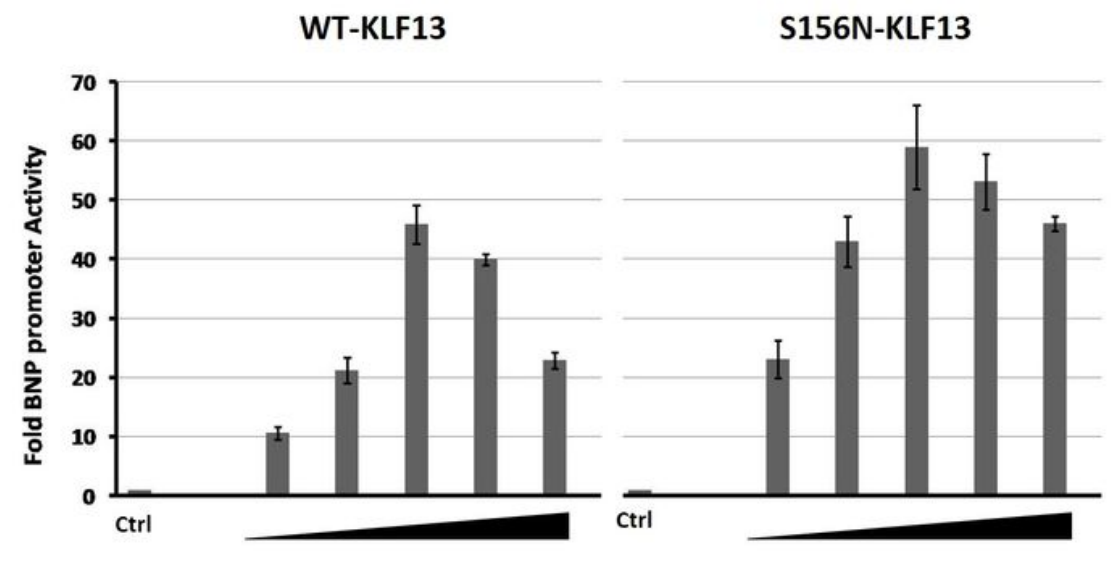

S156N-KLF13

P163S-KLF13

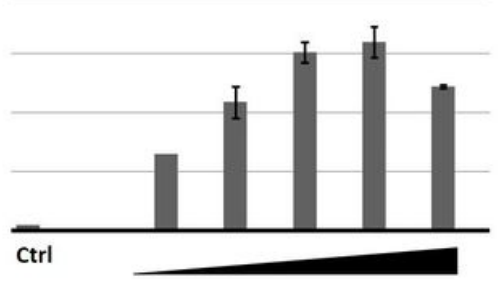

Figure 2

Characterization of KLF13 variants. A: Immunocytochemical staining to detect the cellular localization of KLF13 variants transfected in 293T cells. The green color shows the Alexa 488 conjugated secondary antibodies against the anti-myc antibody. The nuclei were visualized with DAPI. B: Western blotting showing the expression of the KLF13 variants. C: The transcriptional activities of KLF13 variants on BNP promoter. Transfections were carried out using the human B-type natriuretic peptide (BNP) promoter and increasing dosages $(25,50,100,200$, and $300 \mathrm{ng})$ of expression vectors. Each dosage was conducted in duplicate and the experiment was conducted twice. 
A

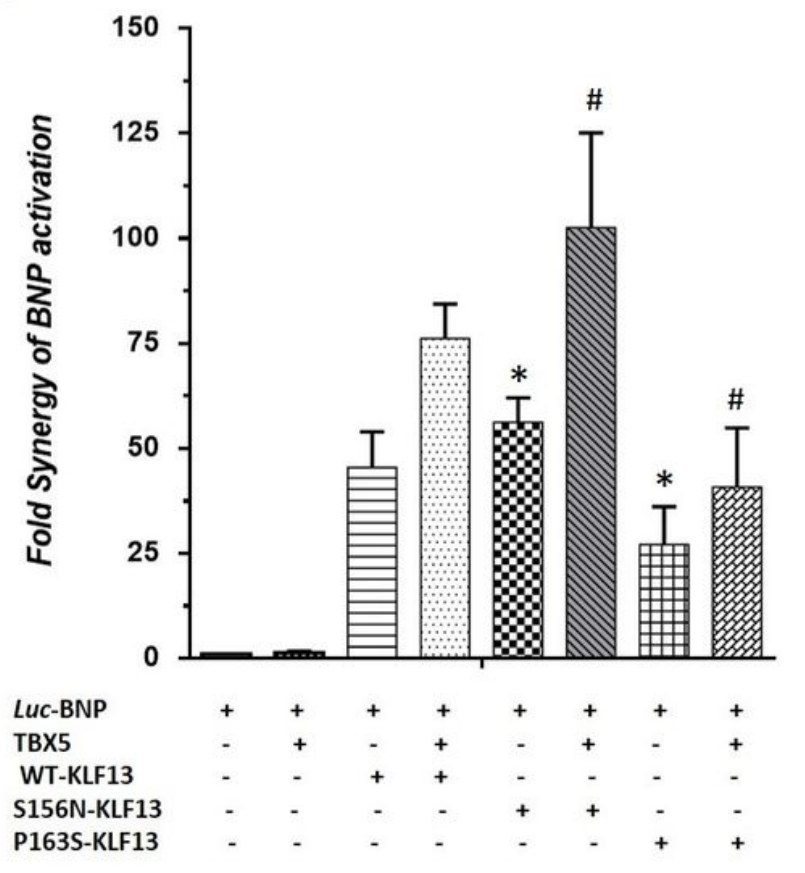

B

$\begin{array}{llll}\text { TBX5 } & + & + & + \\ \text { WT-KLF13 } & + & - & - \\ \text { S156N-KLF13 } & - & + & - \\ \text { P163S-KLF13 } & - & - & +\end{array}$

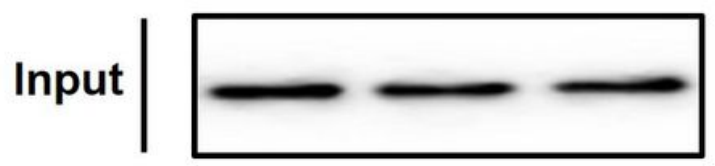

myc

(49kD)

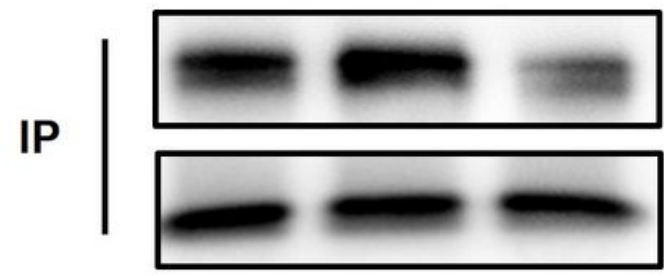

TBX5

$(58 \mathrm{kD})$

myc

(49kD)

\section{Figure 3}

KLF13 variants have effects on genetic interaction with TBX5. A: Luciferase activity in NIH3T3 cells cotransfected with wild-type KLF13 or mutant-expression constructs and the BNP promoter, showing that the P163S mutant could decrease the synergistic activation of the BNP promoter, while the S156N mutant increased the synergistic activation of BNP.B: Coimmunoprecipitations were carried out using 293T cells expressing Myc-KLF13 and TBX5 proteins in combination. The top panel shows Western blots of the nuclear extracts used, with the indicated antibodies. The bottom panel is a Western blot of the anti-TBX5 immunoprecipitates. ( ${ }^{*} p<0.05$, Statistical significant vs WT, \# $p<0.05$, Statistical significant vs WT cotransfected with TBX5).

\section{Supplementary Files}

This is a list of supplementary files associated with this preprint. Click to download.

- Table2.pdf

- Table1.pdf 